\title{
OPERATOR MODELS AND ARVESON'S CURVATURE INVARIANT
}

\author{
MIROSLAV ENGLIŠ \\ MÚ $A V \check{C} R$, Žitná 25 \\ 11567 Prague 1, Czech Republic \\ E-mail: englis@math.cas.cz
}

1. Introduction. In a series of three recent papers [Ar1]-[Ar3], W. Arveson studied tuples of commuting operators $T=\left(T_{1}, \ldots, T_{d}\right)$ satisfying $I-T_{1} T_{1}^{*}-\cdots-T_{d} T_{d}^{*} \geq 0$. Among other things, he established a kind of dilation theorem for such tuples, and introduced a certain so-called "curvature invariant", as well as Euler characteristic, Dirac operator, etc. In this talk, we will discuss possible extensions of his theory to the more general case of commuting tuples satisfying $p\left(T, T^{*}\right) \geq 0$, where $p(x, \bar{y})$ is a polynomial such that $1 / p$ is positive definite and $p\left(T, T^{*}\right):=p\left(L_{T}, R_{T^{*}}\right) I$, where $L_{T}$ is the tuple of the left multiplications by $T_{j}$, and similarly $R_{T^{*}}$ are the right multiplications by $T_{j}^{*}$. Basically, it turns out that everything works fine as long as $p$ satisfies certain additional assumptions, namely, that $1 / p$ is a complete NP-kernel; while, on the other hand, without this assumption it may happen that even the most basic ingredient of Arveson's theory, the curvature invariant, either does not exist or exists but is no longer an integer. Thus Arveson's theory is seen to be something which is peculiar only to the complete Nevanlinna-Pick kernels.

2. Arveson's theory for the ball. Let us briefly recall what Arveson's theory is about. Let $T=\left(T_{1}, \ldots, T_{d}\right)$ be a commuting $d$-tuple of operators on an arbitrary separable Hilbert space $H$ satisfying

$$
\sum_{j} T_{j} T_{j}^{*} \leq I
$$

2000 Mathematics Subject Classification: Primary 47A45; Secondary 47A13, 46E22, 47A15.

Key words and phrases: coanalytic model, reproducing kernel, commuting operator tuple, curvature invariant, majorization function.

Research supported by GA AV ČR grant no. A1019304.

The paper is in final form and no version of it will be published elsewhere. 
Such tuples are called row contractions (since the condition is equivalent to the row operator $\left[T_{1}, \ldots, T_{d}\right]$ being a contraction); following [Ar1]-[Ar3], we will call such tuples simply contractive. In terms of the operator transform

$$
\phi(X):=T_{1} X T_{1}^{*}+\cdots+T_{d} X T_{d}^{*},
$$

this may be rephrased as $I \geq \phi(I)$. Tuples $T$ satisfying in addition

$$
\phi^{m}(I) \rightarrow 0 \quad(\mathrm{SOT}) \quad \text { as } m \rightarrow \infty
$$

are called pure. (For $d=1$, the tuple $T=\left(T_{1}\right)$ consists just of a single operator, its contractivity is equivalent to the ordinary contractivity $\left\|T_{1}\right\| \leq 1$ of $T_{1}$, and $T$ is pure iff $T_{1}^{* n} \rightarrow 0$ strongly.) Denoting further

$$
\Delta:=\left(I-\sum_{j} T_{j} T_{j}^{*}\right)^{1 / 2},
$$

we will say that the tuple $T$ is of finite rank if rank $\Delta<\infty$.

Throughout the rest of this section, let us thus consider a commuting tuple $T$ which is contractive, pure, and of finite rank.

An elementary argument (Schwarz inequality) then shows that $\left\|\sum_{j} \bar{z}_{j} T_{j}\right\|<1$, for any $z \in \mathbf{B}^{d}$. Hence the operator

$$
1-\langle T, z\rangle:=1-\sum_{j} \bar{z}_{j} T_{j}
$$

is invertible, and so is its adjoint $1-\langle z, T\rangle$. We now define, in turn,

$$
\begin{aligned}
F(z) & :=\Delta(1-\langle z, T\rangle)^{-1}(1-\langle T, z\rangle)^{-1} \Delta, \\
\kappa(z) & :=\left(1-|z|^{2}\right) \operatorname{tr} F(z), \\
\kappa(\zeta) & :=\lim _{z \rightarrow \zeta \text { nontangentially }} \kappa(z), \\
\kappa & :=\int_{\partial \mathbf{B}^{d}} \kappa(\zeta) d \sigma(\zeta) .
\end{aligned}
$$

The last quantity $\boldsymbol{\kappa}$ is called the curvature invariant.

The two main results of [Ar2] are the following. First of all, $\kappa(\zeta)$ and $\kappa$ are well defined (i.e. the limits and the integral exist). Second, $\kappa$ is in fact an integer between 0 and $\operatorname{rank}(\Delta)$ (this was verified in [Ar2] only under certain additional hypotheses).

The fact that $\boldsymbol{\kappa}$ is an integer is somewhat surprising, and raises the question whether this does not have some deeper reason-for instance, whether $\boldsymbol{\kappa}$ cannot be interpreted as an index of some operator, or the dimension of some space, or some similar quantity which can only be an integer.

It was therefore conjectured in [Ar2], and proved again under some additional hypotheses, that $\boldsymbol{\kappa}$ coincides with the so-called Euler characteristic of $T$, defined as follows. The space $H$ can be viewed as a module over the ring of polynomials $\mathbf{C}\left[z_{1}, \ldots, z_{d}\right]$ by defining

$$
z_{j} \cdot f:=T_{j} f
$$

By a theorem of Hilbert, there exists a finite free resolution of $H$, i.e. an exact sequence of $\mathbf{C}\left[z_{1}, \ldots, z_{d}\right]$-modules

$$
0 \rightarrow M_{m} \rightarrow M_{m-1} \rightarrow \cdots \rightarrow M_{2} \rightarrow M_{1} \rightarrow H \rightarrow 0
$$


where each $M_{j}=\oplus^{\beta_{j}} \mathbf{C}\left[z_{1}, \ldots, z_{d}\right]$. The alternating sum

$$
\chi(T):=\sum_{j}(-1)^{j} \beta_{j}
$$

is independent of the resolution. This is the Euler characteristic. There is also an equivalent definition: for large $n$, the dimension

$$
\delta(n):=\operatorname{dim}\{\psi \cdot h: \operatorname{deg} \psi \leq n, h \in \operatorname{Ran} \Delta\}
$$

can be shown to be a polynomial in $n$, called the Hilbert polynomial. Its leading term is $\frac{\chi(T)}{d !} n^{d}$.

The "additional hypothesis", alluded to above twice, is that the tuple $T$ be graded, meaning that there exists a continuous representation $\epsilon \mapsto U_{\epsilon}$ of the circle group $\mathbf{T}:=$ $\{\epsilon \in \mathbf{C}:|\epsilon|=1\}$ in the unitary operators on $H$ such that

$$
U_{\epsilon}^{*} T U_{\epsilon}=\epsilon T \quad \forall \epsilon \in \mathbf{T} .
$$

Similarly, in his later paper [Ar3], Arveson proves that (again under some additional hypothesis) $\boldsymbol{\kappa}$ can be interpreted as the index of a certain (Fredholm) operator. Namely, he considers a certain Koszul complex (essentially the same one as in the definition of the familiar Taylor spectrum) and defines a Dirac operator $D_{+}$acting on it. (The exact definition of $D_{+}$involves Clifford numbers and other technicalities, and therefore will not be discussed here.) He then proves that if $T$ is graded, then the kernels of both $D_{+}$and $D_{+}^{*}$ are finite dimensional, and $\boldsymbol{\kappa}=(-1)^{d}\left(\operatorname{dim} \operatorname{ker} D_{+}-\operatorname{dim} \operatorname{ker} D_{+}^{*}\right)$. (So if $D_{+}$has also closed range, then $D_{+}$is Fredholm and $\boldsymbol{\kappa}=(-1)^{d}$ ind $\left.D_{+} \cdot\right)$

In summary, Arveson's results thus are:

(A1) the boundary values $\kappa(\zeta)$ and their integral $\kappa$ exist, and $0 \leq \kappa \leq \operatorname{rank} \Delta$;

(A2) $\kappa$ is always an integer (for $T$ graded);

(A3) $\kappa=\chi \quad$ (for $T$ graded);

(A4) $\boldsymbol{\kappa}=(-1)^{d}\left(\operatorname{dim} \operatorname{ker} D_{+}-\operatorname{dim} \operatorname{ker} D_{+}^{*}\right)$

(for $T$ graded).

Subsequently, there has been a lot of effort to prove the above results in full generality, i.e. without the gradedness hypothesis in (A2)-(A4). For (A2), this was done by Greene, Richter and Sundberg [GRS]. For (A3), it turned out that the general assertion was false (the counterexample was in fact furnished by Arveson himself, see Proposition 7.4 in [Ar2]). The item (A4) was resolved completely for the simplest case of $d=1$, i.e. when the tuple $T$ consists of a single operator which is contractive $(\|T\| \leq 1)$, satisfies $T^{* n} \rightarrow 0$ (SOT) and is such that $I-T T^{*}$ has finite rank. Then it was shown by S. Parrott $[\mathrm{Pa}]$, with a later simplification by R.N. Levy [Le], that in this case the definition of $\boldsymbol{\kappa}$ can be rephrased as

$$
\kappa=\lim _{n \rightarrow \infty} \operatorname{tr}\left(T^{n} T^{* n}-T^{n+1} T^{* n+1}\right)
$$

(the existence of the trace being a consequence of the hypotheses on $T$ ), and Arveson's result for the Dirac operator from the last paragraph translates into

$$
\kappa=\operatorname{dim} \operatorname{Ran}\left(I-T T^{*}\right)-\operatorname{dim} \operatorname{Ran}\left(I-T^{*} T\right)=-\operatorname{ind} T,
$$

without any gradedness/closed range hypothesis. (And, again, the fact that $T$ is Fredholm - so that ind $T$ is well defined - is part of the assertion.) 
In the general case $(d>1)$, the problem (A4) remains unsolved.

All the theory just described hinges on another result of Arveson, which appeared in [Ar1], the first of the three papers mentioned above, and which actually describes a model for a general contractive tuple:

Consider the space $\mathcal{H}^{1}\left(\mathbf{B}^{d}\right)$, the Hilbert space of holomorphic functions on the unit ball $\Omega=\mathbf{B}^{d}$ whose reproducing kernel is $K(x, \bar{y})=1 /(1-\langle x, y\rangle)$. That is, $\mathcal{H}^{1}$ consists of all holomorphic functions $f(z)=\sum_{\alpha \text { multiindex }} f_{\alpha} z^{\alpha}$ on $\mathbf{B}^{d}$ such that

$$
\|f\|^{2}:=\sum_{\alpha} \frac{\alpha !}{|\alpha| !}\left|f_{\alpha}\right|^{2}<\infty,
$$

equipped with the norm $\|f\|$ and the corresponding scalar product. (For $d=1$, this reduces just to the familiar Hardy space $H^{2}$, and $\langle f, g\rangle$ can be expressed as the integral of the boundary values of $f \bar{g}$ over the unit circle; in higher dimensions, however, no such integral representation is possible - the best one can do is to interpret $\mathcal{H}^{1}$ as a certain Besov-type space.)

Further, denote by $Z=\left(Z_{1}, \ldots, Z_{d}\right)$, where

$$
Z_{j}: f(z) \mapsto z_{j} f(z),
$$

the commuting tuple of multiplications by the coordinate functions $z_{1}, \ldots, z_{d}$ on $\mathcal{H}^{1}$.

Then the following is true:

TheOREM ([Ar1]). For any pure contractive tuple $T$ there exists an auxiliary separable Hilbert space $\mathcal{E}$ and an isometry $V: H \rightarrow \mathcal{H} \otimes \mathcal{E}$ such that

$$
V T^{*}=\left(Z^{*} \otimes I\right) V \text {. }
$$

(That is, $T^{*}$ is unitarily equivalent to the restriction of $Z^{*} \otimes I$ to an invariant subspace.)

In fact, one can take $\mathcal{E}=\overline{\operatorname{Ran} \Delta}$ (so if $T$ is of finite rank, $\mathcal{E}$ can be taken finite dimensional.)

In this talk, we investigate the possibility of extending the first two of Arveson's results - i.e. on the existence and the integrality of $\kappa$ - to operator tuples satisfying, instead of the row contractivity

$$
I-T_{1} T_{1}^{*}-\cdots-T_{d} T_{d}^{*} \geq 0
$$

a condition of a more general type, namely

$$
p\left(T, T^{*}\right) \geq 0
$$

where $p(x, \bar{y})$ is a polynomial in $x$ and $\bar{y}$, and $p\left(T, T^{*}\right)$ is obtained by substituting $T^{\alpha} T^{* \beta}$ for $x^{\alpha} \bar{y}^{\beta}$. Our main result is that all works fine under a suitable additional hypothesis on $p$ (which, however, seems to be satisfied not too often), while, on the other hand, there are some very natural choices of $p$ for which even (A1) or (A2) fail to hold.

3. Operator models. We begin by recalling the general analogue, proved in $[\mathrm{AEM}]$, of the above model theorem for contractive tuples. Recall that a function $K(x, \bar{y})$ of two variables $x, y \in \Omega$, where $\Omega$ is a domain in $\mathbf{C}^{d}$, is called positive definite if for any integer 
$n \geq 0$ and any points $x_{1}, \ldots, x_{n} \in \Omega$ and numbers $c_{1}, \ldots, c_{n} \in \mathbf{C}$,

$$
\sum_{i, j=1}^{n} K\left(x_{i}, \bar{x}_{j}\right) c_{i} \bar{c}_{j} \geq 0 \text {. }
$$

It is a theorem of Aronszajn [An] that for any positive definite function there exists a unique (up to isomorphism) Hilbert space $\mathcal{H}_{K}$ of functions on $\Omega$ which has $K(x, \bar{y})$ for its reproducing kernel, i.e. $K_{x}:=K(\cdot, \bar{x}) \in \mathcal{H}_{K} \forall x \in \Omega$ and

$$
f(x)=\left\langle f, K_{x}\right\rangle \quad \forall f \in \mathcal{H}_{K} .
$$

From now on, we assume that $p(x, y)$ is a polynomial such that

(a) $K(x, \bar{y}):=\frac{1}{p(x, \bar{y})}$ is positive definite;

(b) the Hilbert space $\mathcal{H}_{K}$ contains the polynomials, and they are dense in it;

(c) the operator tuple $Z=\left(Z_{1}, \ldots, Z_{d}\right)$ of multiplications by the coordinate functions maps $\mathcal{H}_{K}$ (boundedly) into itself.

Owing to (b), there exists an orthonormal basis $\left\{\psi_{j}\right\}_{j=0}^{\infty}$ of $\mathcal{H}_{K}$ consisting of polynomials (to see this, start with the monomials and apply the usual Gram-Schmidt process). Fix such a basis from now on, and let

$$
f_{m}(x, \bar{y}):=1-\sum_{j=0}^{m-1} \psi_{j}(x) p(x, \bar{y}) \overline{\psi_{j}(y)} .
$$

Since this is a polynomial, we can, for any commuting tuple $T=\left(T_{1}, \ldots, T_{d}\right)$ of operators on another Hilbert space $H$, form $f_{m}\left(T, T^{*}\right)$ in the same way as $p\left(T, T^{*}\right)$, i.e. by writing all $T$ 's to the left of all $T^{*}$ 's; in other words,

$$
f_{m}\left(T, T^{*}\right):=f_{m}\left(L_{T}, R_{T^{*}}\right) I
$$

where $L_{T}, R_{T^{*}}$ is the commuting $2 d$-tuple of operators on $\mathcal{B}(H)$ given by $L_{T_{j}}: X \mapsto$ $T_{j} X$ and $R_{T_{j}^{*}}: X \mapsto X T_{j}^{*}, j=1, \ldots, d$. Then we have the following "model theorem" from $[\mathrm{AEM}]$.

TheOrem $([\mathrm{AEM}])$. For any commuting tuple $T=\left(T_{1}, \ldots, T_{d}\right)$ on $H$ satisfying

$$
\begin{aligned}
& p\left(T, T^{*}\right) \geq 0, \\
& f_{m}\left(T, T^{*}\right) \rightarrow 0 \quad(\mathrm{SOT}),
\end{aligned}
$$

there exists an auxiliary Hilbert space $\mathcal{E}$ and an isometry $V: H \rightarrow \mathcal{H} \otimes \mathcal{E}$ such that

$$
V T^{*}=\left(Z^{*} \otimes I\right) V \text {. }
$$

In fact, one can take $\mathcal{E}=\overline{\operatorname{Ran} \Delta}$, where

$$
\Delta:=p\left(T, T^{*}\right)^{1 / 2} .
$$

By analogy with Arveson's terminology, we will call the tuples $T$ satisfying (1) $p$ contractive, those satisfying (2) ( $p$-)pure, and those for which $\operatorname{rank} \Delta<\infty$ of $(p$-)finite rank.

Arveson's original space $\mathcal{H}^{1}\left(\mathbf{B}^{d}\right)$ and his row contractions are obtained upon choosing $p(x, \bar{y})=1-\langle x, y\rangle$. 
The operator $V$ above is explicitly given by

$$
V h=\sum_{j=0}^{\infty} \psi_{j} \otimes \Delta \psi_{j}(T)^{*} h, \quad h \in H
$$

(the convergence of the sum is a consequence of (2); see [AEM]). Note that if we interpret (as we may) the elements of $\mathcal{H} \otimes \mathcal{E}$ as $\mathcal{E}$-valued functions on $\Omega$, then (3) becomes simply

$$
V h(z)=\Delta K(z, T) h \in \mathcal{E} .
$$

The adjoint of $V$ satisfies

$$
V^{*}(f \otimes h)=f(T) \Delta h,
$$

for any polynomial $f \in \mathcal{H}$ and $h \in \mathcal{E}$.

4. Arveson's theory for $p$-contractive tuples. Let us now try to follow step by step the arguments of Arveson, and see what obstacles occur. Thus let $p(x, \bar{y})$ be a polynomial satisfying the assumptions (a)-(c) from Section $3, \mathcal{H}=\mathcal{H}_{K}$ the corresponding reproducing kernel Hilbert space $(K=1 / p)$, and $T=\left(T_{1}, \ldots, T_{d}\right)$ a commuting $d$-tuple of operators on a Hilbert space $H$ which is $p$-contractive and $p$-pure (i.e. (1) and (2) hold), and let us see what we can do.

The first obstacle is encountered right at the beginning, when defining the functions

$$
F(z)=\Delta(1-\langle z, T\rangle)^{-1}(1-\langle T, z\rangle)^{-1} \Delta, \quad \kappa(z):=\left(1-|z|^{2}\right) \operatorname{tr} F(z) .
$$

Plainly, their counterparts in the general case should be

$$
F(z)=\Delta p(z, T)^{-1} p(T, z)^{-1} \Delta, \quad \kappa(z):=p(z, z) \operatorname{tr} F(z) .
$$

However, in general there seems to be no reason at all why the inverse $p(T, z)^{-1}$ that is, $K(T, z)$-should exist. Fortunately, there is a simple solution to this, provided by our operator model constructed in the previous section. Recall that it asserts that there is an isometry $V: H \rightarrow \mathcal{H} \otimes \mathcal{E}$ such that

$$
V T^{*}=\left(Z^{*} \otimes I\right) V .
$$

Using this (cf. (4)), the function $F(z)$ above is seen to satisfy

$$
\langle F(z) h, h\rangle=\|K(T, \bar{z}) \Delta h\|^{2}=\left\|V^{*}\left(K_{z} \otimes h\right)\right\|^{2} .
$$

Thus

$$
\kappa(z)=p(z, z) \operatorname{tr} F(z)=\frac{\operatorname{tr}\left\langle V V^{*}\left(K_{z} \otimes \cdot\right),\left(K_{z} \otimes \cdot\right)\right\rangle}{K(z, z)} .
$$

REMARK. Observe that $\kappa(z)$ is a kind of Berezin transform: indeed, omitting the tensor products, we get

$$
\frac{\left\langle V V^{*} K_{z}, K_{z}\right\rangle}{\left\langle K_{z}, K_{z}\right\rangle}
$$

which is the usual definition of the Berezin transform of the operator $V V^{*}$.

We can thus skip the definitions of $K(T, \bar{z})$ and $F(z)=\Delta K\left(z, T^{*}\right) K(T, \bar{z}) \Delta$ and instead define $\kappa(z)$ directly by the formula (5). Though this formula involves the isometry $V$ from the model theorem, it can be shown to be independent of the possible freedom in 
the choice of such $V$, and the function $\kappa(z)$ is thus defined unambiguously and depends only on $T$.

The second, and much more serious, obstacle is the existence of the boundary values of $\kappa(z)$. Let us recall how Arveson's proof of this proceeds (in the language of the present paper). Since $V$ is an isometry, $V V^{*}$ is the orthogonal projection onto its range Ran $V$ in $\mathcal{H} \otimes \mathcal{E}$. Hence $P=I-V V^{*}$ is the projection onto the $(Z \otimes I)$-invariant subspace $\mathcal{M}:=(\operatorname{Ran} V)^{\perp}$.

At this moment, Arveson proceeds by using the following remarkable fact: any such $P$ can be factored as $P=\Phi \Phi^{*}$, where $\Phi$ is a mapping from $\mathcal{H} \otimes E$ into $\mathcal{H} \otimes \mathcal{E}$, for some auxiliary space $E$, satisfying

$$
\Phi(Z \otimes I)=(Z \otimes I) \Phi .
$$

Alternatively, any such $\Phi$ may be viewed as a holomorphic operator-valued function $\Phi: \Omega \rightarrow \mathcal{B}(E, \mathcal{E})$ satisfying

$$
\|\Phi(x)\| \leq 1 \quad \forall x \in \Omega .
$$

Feeding this information back into the formula for $\kappa(z)$, we obtain

$$
\kappa(z)=\operatorname{tr}\left[I-\Phi(z) \Phi(z)^{*}\right]
$$

and the existence of boundary values follows in view of the boundedness of $\Phi$ and Fatou's theorem. Integrating, we also see that $\boldsymbol{\kappa}$ (exists and) is nonnegative and not greater than $\operatorname{rank}(\Delta)$.

Returning to the general case of $p$-contractive tuples, we are thus lead to the question whether, for any Hilbert space $\mathcal{E}$ and any $(Z \otimes I)$-invariant subspace $\mathcal{M}$ of $\mathcal{H} \otimes \mathcal{E}$, the projection onto $\mathcal{M}$ can always be factored as

$$
P_{\mathcal{M}}=\Phi \Phi^{*}
$$

with some $\Phi: \mathcal{H} \otimes E \rightarrow \mathcal{H} \otimes \mathcal{E}$ satisfying (6).

There is a simple necessary condition for the existence of such factorization. Assume, for simplicity, that $K(\cdot, 0)=\mathbf{1}$ (this is the case in most cases of interest anyway), and take $\mathcal{E}=\mathbf{C}$ and $\mathcal{M}=\{f: f(0)=0\} \subset \mathcal{H} \simeq \mathcal{H} \otimes \mathbf{C}$. Then $I-P_{\mathcal{M}}=\langle\cdot, \mathbf{1}\rangle \mathbf{1}$, so that

$$
\left\langle P_{\mathcal{M}} K_{y}, K_{x}\right\rangle=\left\langle(I-\langle\cdot, \mathbf{1}\rangle \mathbf{1}) K_{y}, K_{x}\right\rangle=K(x, \bar{y})-1 .
$$

Hence $P_{\mathcal{M}}=\Phi \Phi^{*}$ implies

$$
K(x, \bar{y})-1=\left\langle\Phi^{*} K_{y}, \Phi^{*} K_{x}\right\rangle=\Phi(x) \Phi(y)^{*} K(x, \bar{y}),
$$

or $1-1 / K(x, \bar{y})=\Phi(x) \Phi(y)^{*}$. Consequently,

$$
1-\frac{1}{K(x, \bar{y})} \quad \text { is a positive definite function. }
$$

Kernels $K(x, \bar{y})$ with this property are called complete NP-kernels.

Surprisingly, this condition turns out to be sufficient as well:

Theorem (McCullough and Trent [MT]). If $\mathcal{H}$ has complete $N P$-kernel and $\mathcal{M} \subset \mathcal{H} \otimes \mathcal{E}$ is a subspace invariant under $(Z \otimes I)$, then the projection $P$ onto $\mathcal{M}$ factors as $P=\Phi \Phi^{*}$ with $\Phi$ as in (6).

Consequently, Arveson's curvature invariant $\boldsymbol{\kappa}$ exists in this case. 
We indicate the proof for the case $\mathcal{E}=\mathbf{C}($ so $\mathcal{M} \subset \mathcal{H})$. From the condition that $K$ be a complete NP-kernel it follows that there exist holomorphic functions $\phi_{j}(j=1,2, \ldots)$ on $\Omega$ such that

$$
1-1 / K(x, \bar{y})=\sum_{j} \phi_{j}(x) \overline{\phi_{j}(y)} .
$$

Since $\mathcal{M}$ is $Z$-invariant, $Z P=P Z P$ and $P Z^{*}=P Z^{*} P$; hence also $P \phi(Z)^{*}=P \phi(Z)^{*} P$. (See e.g. Lemma 4.1 in [GRS].) Thus

$$
\begin{aligned}
\left\langle\left(P-\sum_{j} \phi_{j}(Z) P \phi_{j}(Z)^{*}\right) f, f\right\rangle & =\|P f\|^{2}-\sum_{j}\left\|P \phi_{j}(Z)^{*} f\right\|^{2} \\
& =\|P f\|^{2}-\sum_{j}\left\|P \phi_{j}(Z)^{*} P f\right\|^{2} \\
& \geq\|P f\|^{2}-\sum_{j}\left\|\phi_{j}(Z)^{*} P f\right\|^{2} \\
& =\left\langle\left(I-\sum_{j} \phi_{j}(Z) \phi_{j}(Z)^{*}\right) P f, P f\right\rangle \\
& =\left\langle\frac{1}{K}\left(Z, Z^{*}\right) P f, P f\right\rangle=|\langle P f, \mathbf{1}\rangle|^{2} \geq 0,
\end{aligned}
$$

since $\frac{1}{K}\left(Z, Z^{*}\right)=\langle\cdot, \mathbf{1}\rangle \mathbf{1}$. Thus $X:=P-\sum_{j} \phi_{j}(Z) P \phi_{j}(Z)^{*}$ is a nonnegative operator. Set $E:=\operatorname{Ran} X^{1 / 2}$ and define $\Phi: \mathcal{H} \otimes E \rightarrow \mathcal{H}$ by

$$
\Phi^{*} K_{x}:=K_{x} \otimes X^{1 / 2} K_{x} \quad \text { on } \operatorname{span}\left\{K_{x} ; x \in \Omega\right\} \text {. }
$$

Then $\Phi^{*}$ intertwines $Z^{*}$ and $Z^{*} \otimes I$ (since it preserves their eigenvectors) and

$$
\begin{aligned}
\left\langle\Phi^{*} K_{y}, \Phi^{*} K_{x}\right\rangle & =K(x, \bar{y})\left\langle X K_{y}, K_{x}\right\rangle \\
& =K(x, \bar{y})\left(\left\langle P K_{y}, K_{x}\right\rangle-\sum_{j}\left\langle P \phi_{j}(Z)^{*} K_{y}, \phi_{j}(Z)^{*} K_{x}\right\rangle\right) \\
& =K(x, \bar{y})\left\langle P K_{y}, K_{x}\right\rangle\left(1-\sum_{j} \phi_{j}(x) \overline{\phi_{j}(y)}\right) \\
& =K(x, \bar{y})\left\langle P K_{y}, K_{x}\right\rangle \cdot 1 / K(x, \bar{y})=\left\langle P K_{y}, K_{x}\right\rangle,
\end{aligned}
$$

showing that $\Phi \Phi^{*}$ extends to a bounded operator equal to $P$.

Using the methods of [GRS], one can also prove under mild additional assumptions that $\boldsymbol{\kappa}$ is an integer.

Thus, for complete NP-kernels, all goes well with Arveson's theory. Unfortunately, it turns out that not too many interesting spaces have complete NP-kernels. For instance, for the weighted Bergman spaces $\mathcal{H}^{\nu}(\Omega)$ on bounded symmetric domains, this happens only if $\Omega=\mathbf{B}^{d}$ and $0<\nu \leq 1$. Similarly, for the (unweighted) Bergman space on a domain in $\mathbf{C}, 1-\frac{1}{K}$ fails to be positive definite unless the domain is simply connected, because on multiply connected domains $K$ is known to always have a zero!

So, what can be done for non-NP kernels? 
Unfortunately, results are lacking. In [En], it was shown that for kernels $K(x, \bar{y})$ having the form

$$
\frac{1}{K(x, \bar{y})}=\left(b-\sum_{j} b_{j}(x) \overline{b_{j}(y)}\right)-\left(c-\sum_{j} c_{j}(x) \overline{c_{j}(y)}\right),
$$

for suitable real constants $b, c$ and holomorphic polynomials $b_{j}, c_{j}(j=1,2, \ldots)$ such that both $K \cdot\left(b-\sum_{j} b_{j}(x) \overline{b_{j}(y)}\right)$ and $K \cdot\left(c-\sum_{j} c_{j}(x) \overline{c_{j}(y)}\right)$ are positive definite, one can at least show that any projection $P$ onto a $(Z \otimes I)$-invariant subspace in $\mathcal{H} \otimes \mathcal{E}$ can be expressed as

$$
P=\Phi\left[I-\Gamma \Gamma^{*}\right] \Phi^{*},
$$

where $\Gamma, \Phi$ are operator-valued holomorphic functions satisfying (6), and $\|\Gamma(x)\| \leq 1$ and $\|\Phi(x)\| \leq C K(x, x)^{1 / 2}$. (Note that complete NP-kernels are a special case of $(7)$ corresponding to $c_{j}=0$ for all $j, c=0$ and $b=1$. It was shown in [En] that both the Szegö kernel and the standard weighted Bergman kernels on $\mathbf{B}^{d}$, and even on any bounded symmetric domain, have the form (7).) Unfortunately, as $\Phi$ is unbounded, hence need not have boundary values, this is insufficient to deduce the existence of the boundary values of $\kappa(x)$.

We now show that this insufficiency is not a defect of the method, but lies at the heart of the matter: namely, we exhibit a situation in which $\kappa(z)$ either fails to have the boundary values, or does have them but then $\boldsymbol{\kappa}$ is not an integer. In other words, either (A1) or (A2) from Arveson's results fail. Consequently, no general analogue of Arveson's theory has a chance to exist. Surprisingly, this is the case even for the very simple example of the ordinary Bergman space on the unit disc.

Finally, we remark that one more way to possibly circumvent the existence of the boundary values of $\kappa$ would be to look directly at the limit

$$
\boldsymbol{\kappa}^{*}:=\lim _{r \nearrow^{1}} \int_{\partial \mathbf{B}^{d}} \kappa(r \zeta) d \sigma(\zeta)
$$

(This is for the case of the unit ball $\Omega=\mathbf{B}^{d}$; for general domains, one might consider integrals over an appropriate family of surfaces tending to $\partial \Omega$.) Indeed, as $\kappa$ is always bounded on $\Omega$ (by $\operatorname{rank}(\Delta)$ - this follows easily from (5)), it is immediate from the Lebesgue Bounded Convergence Theorem that if (A1) holds, then $\boldsymbol{\kappa}^{*}$ exists and equals $\boldsymbol{\kappa}$. Clearly, it could happen that $\boldsymbol{\kappa}^{*}$ exists even if $\kappa$ fails to have boundary values on some piece of the boundary of positive measure.

Unfortunately, we will show that even this approach suffers from the same deficiency as above: namely, it may again happen that $\boldsymbol{\kappa}^{*}$ either fails to exist, or exists but is not an integer. Let us now proceed to the details.

5. A counterexample. Let $\mathbf{D}$ be the unit disc in $\mathbf{C}$ and $p(x, \bar{y})=(1-x \bar{y})^{2}$. Then $K=1 / p$ is a positive definite function and the corresponding Hilbert space $\mathcal{H}_{K}$ is just the standard Bergman space $L_{a}^{2}(\mathbf{D})$ of square-integrable holomorphic functions on $\mathbf{D}$. Let $Z$ be the operator of multiplication by $z$ on $L_{a}^{2}(\mathbf{D})$, and let $\mathcal{M}$ be a $Z$-invariant subspace of $L_{a}^{2}(\mathbf{D})$ and $P_{\mathcal{M}}$ the projection onto $\mathcal{M}$. By our model theorem from Section $3, Z^{*}$ is 
a model for operators $T^{*}$ satisfying

$$
I-2 T T^{*}+T^{2} T^{* 2} \geq 0 .
$$

(This resembles the 2-contractivity condition of Agler [Ag].)

Let us compute the function $\kappa(z)$ for the operator 1-tuple $T$ on $H$, where $H=\mathcal{M}^{\perp}$ and $T^{*}$ is the restriction of $Z^{*}$ to $H$. One checks easily that $T$ is $p$-contractive, pure, and has rank 1. Clearly, we can take for the isometry $V$ the inclusion operator $H \hookrightarrow L_{a}^{2}(\mathbf{D})$. Thus $V V^{*}=I-P_{\mathcal{M}}$, and by $(5), \kappa(z)$ is simply the Berezin transform of $I-P_{\mathcal{M}}$ :

$$
\kappa(z)=\frac{\left\langle V V^{*} K_{z}, K_{z}\right\rangle}{K(z, z)}=1-\frac{\left\langle P_{\mathcal{M}} K_{z}, K_{z}\right\rangle}{K(z, z)}=1-\left\langle P_{\mathcal{M}} k_{z}, k_{z}\right\rangle,
$$

where $k_{z}:=K_{z} /\left\|K_{z}\right\|$ is the unit vector in the direction of $K_{z}$. Consequently, $\kappa(z)$ has (nontangential) boundary values if and only if the function

$$
k_{\mathcal{M}}(z):=\left\langle P_{\mathcal{M}} k_{z}, k_{z}\right\rangle
$$

has them; and, further (since always $0 \leq k_{\mathcal{M}}(z) \leq 1$ ), if these boundary values $k_{\mathcal{M}}(\zeta$ ) exist then

$$
\boldsymbol{\kappa}=1-\int_{\mathbf{T}} k_{\mathcal{M}}(\zeta) d \sigma(\zeta)
$$

is an integer if and only if these boundary values are either 1 a.e. or 0 a.e.

The function $k_{\mathcal{M}}(z)$ was studied in detail by Aleman, Richter and Sundberg under the name of majorization function in [ARS]. Recall that the extremal function of $\mathcal{M}$ is the (unique) solution to the extremal problem

$$
\max \left\{\operatorname{Re} G^{(k)}(0) ; G \in \mathcal{M},\|G\| \leq 1\right\}
$$

where $k \geq 0$ be the smallest integer for which there exists $f \in \mathcal{M}$ with $f^{(k)}(0) \neq$ 0 . An extremal function is inner (i.e. satisfies $G \perp Z^{k} G \forall k>1$ ); conversely, any inner function $G$ is the extremal function for the $Z$-invariant subspace $[G]$ generated by it (i.e. the closed linear span of $Z^{k} G, k=0,1,2, \ldots$ ). Also, a $Z$-invariant subspace $\mathcal{M}$ is said to be of index one if $\operatorname{dim}(\mathcal{M} \ominus Z \mathcal{M})=1$. It is known that if $\mathcal{M}$ is of index one and $G$ is its extremal function, then $\mathcal{M}=[G]$.

The following remarkable result appears as Corollary 5.9 in [ARS].

TheOrem. Let $\mathcal{M}=[G]$ be an index-one $Z$-invariant subspace of $L_{a}^{2}(\mathbf{D})$ with extremal function $G$. Then there exist two measurable subsets $N$ and $P$ of $\mathbf{T}$ such that $N \cap P$ has measure zero, $N \cup P$ has full measure in $\mathbf{T}$, and

- on $N$, the nontangential limits of $G$ exist, and the nontangential limits of $k_{\mathcal{M}}$ exist and are equal to 1 ;

- at any $\zeta \in P$, the cluster set of $G$ at $\zeta$ is the entire $\mathbf{C}$, and the nontangential lim-inf of $k_{\mathcal{M}}$ at $\zeta$ is 0 .

It is a conjecture that the second part can be completed by "... and the nontangential lim-sup is 1 ", i.e. that the nontangential limits of $k_{\mathcal{M}}$ do not exist at any point of $P$. If this is true, then it follows that (for index-one subspaces) the boundary values of $k_{\mathcal{M}}(z)$ exist a.e. on $\mathbf{T}$ if and only if the set $P$ is empty, i.e. if and only if the extremal function $G$ has boundary values a.e. on $\mathbf{T}$ (and in that case, $k_{\mathcal{M}}(\zeta)=1$ a.e. on $\mathbf{T}$, so that $\boldsymbol{\kappa}=0$ ). However, it is known that there exist inner functions $G$ which fail to have boundary values 
on a set of positive measure: it may even happen that $G$ has nontangential boundary values almost nowhere on $\mathbf{T}$ ! Consequently, it would follow that there exist $Z$-invariant subspaces $\mathcal{M}$ for which $k_{\mathcal{M}}(z)$ fails to have boundary values at a.a. points of $\mathbf{T}$, implying that Arveson's (A1) fails drastically.

Unfortunately, the above conjecture remains still only a conjecture as of this writing. We therefore exhibit, at least, an example in which the nontangential boundary values either do not exist, or if they do, then the curvature $\kappa$ turns out to be a noninteger (and, in fact, can assume any value between 0 and 1).

For a function $f \in L_{a}^{2}(\mathbf{D})$, its "zero-set" is defined to be the sequence of all the zeroes of $f$ in $\mathbf{D}$, each zero being repeated according to its multiplicity. (So the zero-set is not really a set, but rather a sequence; however, the terminology has already become universally accepted.) Conversely, for any sequence $\Lambda$ of points of $\mathbf{D}$ we denote by $I(\Lambda)$ the subspace of all functions in $L_{a}^{2}(\mathbf{D})$ that vanish at each $\lambda \in \Lambda$, counting multiplicities. Then the space $I(\Lambda)$ either reduces to the constant zero, or is an index-one $Z$-invariant subspace of $L_{a}^{2}(\mathbf{D})$.

We now modify a construction from $[\mathrm{ARS}]$ in order to exhibit the promised counterexample. For typographical reasons, let us write $k[f]$ instead of $k_{[f]}$ for the majorization function of the $Z$-invariant subspace generated by a function $f \in L_{a}^{2}(\mathbf{D})$.

For $\alpha \in \mathbf{D}$, let $B_{\alpha}$ be the Blaschke factor with simple zero at $\alpha$. It is known that (cf. Lemma 6.5 in $[\mathrm{ARS}]$ )

$$
k\left[B_{\alpha}^{m}\right](z)=\left|\frac{z-\alpha}{1-\bar{\alpha} z}\right|^{m}\left(1+m \frac{\left(1-|z|^{2}\right)\left(1-|\alpha|^{2}\right)}{|1-\bar{\alpha} z|^{2}}\right)^{1 / 2} .
$$

Applying this to $\alpha=\left(1-\frac{a}{m}\right)^{1 / n}$, where $a$ is a fixed positive number and $n$ a fixed positive integer, it follows that

$$
\lim _{m \rightarrow \infty} k\left[B_{\sqrt[n]{1-a / m}}^{m}\right](z)=\left(1+2 \frac{a}{n} \frac{1-|z|^{2}}{|1-z|^{2}}\right)^{1 / 2} e^{-\frac{a}{n} \frac{1-|z|^{2}}{|1-z|^{2}}},
$$

uniformly on compact subsets of $\overline{\mathbf{D}} \backslash\{1\}$. Further, it was shown in the proof of Lemma $6.2(\mathrm{~b})$ in [ARS] that the expression on the right-hand side is not greater than $\sqrt{(1+a / 6) e^{-a / 6}}$ if $z=(1-1 / n) e^{i \theta}$ with $|\theta| \leq \pi / n$. Combining this together, and using also rotation invariance, we see that to the given fixed $a, n$ we can always find an integer $M_{a, n}$ such that $\forall m \geq M_{a, n}$,

$$
k\left[B_{\sqrt[n]{1-a / m}}^{m} e^{i \theta_{0}}\right]\left(\left(1-\frac{1}{n}\right) e^{i\left(\theta+\theta_{0}\right)}\right) \leq \sqrt{\frac{1+a / 6}{e^{a / 6}}} \quad \forall|\theta| \leq \frac{\pi}{n}, \forall \theta_{0} \in \mathbf{R} .
$$

Choose now an arbitrary sequence $a_{k}$ of positive numbers tending to infinity (e.g. $a_{k}=k$ ), and denote

$$
\phi_{\delta}(z):=\exp \left(\frac{1}{2 \delta} \int_{-\delta}^{\delta} \frac{e^{i t}+z}{e^{i t}-z} d t\right) .
$$

Then it was shown in [ARS], proof of Theorem 6.6, that there exist $\delta_{k}>0, n_{k} \in \mathbf{N}$, and $m_{k}>M_{a_{k}, n_{k}}$ such that the infinite product $g(z):=\prod_{k=1}^{\infty} \phi_{\delta_{k}}^{a_{k}}\left(z^{n_{k}}\right) B_{1-a_{k} / m_{k}}^{m_{k}}\left(z^{n_{k}}\right)$ converges and defines a (nonzero) function in $L_{a}^{2}(\mathbf{D})$. Let $\Lambda$ be the zero-set of $g$,

$$
\Lambda_{+}:=\Lambda \cap\{z: \operatorname{Im} z \geq 0\}
$$


the portion of $\Lambda$ lying in the closed upper half-plane, and set $\mathcal{M}=I\left(\Lambda_{+}\right)$. Since a subset of an $L_{a}^{2}(\mathbf{D})$-zero set is again an $L_{a}^{2}(\mathbf{D})$-zero set, $\mathcal{M}$ is a nontrivial index-one $Z$-invariant subspace of $L_{a}^{2}(\mathbf{D})$. By construction, $\Lambda_{+}$contains all the zeroes of $B_{1-a_{k} / m_{k}}^{m_{k}}\left(z^{n_{k}}\right)$ lying in the upper halfplane (counting multiplicities), for each $k$; thus

$$
\mathcal{M} \subset\left[B_{\left(1-a_{k} / m_{k}\right)^{1 / n_{k} \exp \left(2 \pi i j / n_{k}\right)}}^{m_{k}}\right], \quad \forall j=0,1, \ldots,\left[\frac{n_{k}}{2}\right] .
$$

Since $\mathcal{M} \subset \mathcal{N}$ clearly implies $k_{\mathcal{M}} \leq k_{\mathcal{N}}$, we thus get from (9)

$$
k_{\mathcal{M}}\left(\left(1-\frac{1}{n_{k}}\right) e^{i\left(\theta+2 \pi / n_{k} j\right)}\right) \leq \sqrt{\frac{1+a_{k} / 6}{e^{a_{k} / 6}}}, \quad \forall|\theta| \leq \frac{\pi}{n_{k}}, \forall j=0, \ldots,\left[\frac{n_{k}}{2}\right], \forall k .
$$

In other words,

$$
k_{\mathcal{M}}\left(\left(1-\frac{1}{n_{k}}\right) \zeta\right) \leq \sqrt{\frac{1+a_{k} / 6}{e^{a_{k} / 6}}} \quad \forall \zeta \in \mathbf{T}^{+}, \forall k,
$$

where we have denoted $\mathbf{T}^{+}:=\mathbf{T} \cap\{z: \operatorname{Im} z \geq 0\}$. As $a_{k}$ tend to infinity by choice, it follows that the nontangential lim-inf of $k_{\mathcal{M}}(z)$ is zero as each point of $\mathbf{T}^{+}$. (In fact, we even have $\liminf \operatorname{in}_{r 1} k_{\mathcal{M}}(r \zeta)=0$ uniformly on $\mathbf{T}^{+}$.)

On the other hand, if $G$ is the extremal function for $\mathcal{M}$, then by results of Carleson and Akutowicz (see e.g. the beginning of Section 7 in [ARS]), $G$ extends holomorphically across $\mathbf{T} \backslash \overline{\Lambda_{+}}$, i.e. across $\mathbf{T}^{-}:=\mathbf{T} \backslash \mathbf{T}^{+}$. By Proposition 5.1 in $[$ ARS], it therefore follows that the nontangential (even unrestricted) limit of $k_{\mathcal{M}}(z)$ equals 1 at each point of $\mathbf{T}^{-}$.

Consequently, one of the following two possibilities must now take place.

(A) There exists a set $E \subset \mathbf{T}^{+}$of positive measure on which the nontangential limits of $k_{\mathcal{M}}$ fail to exist.

Then, since $\kappa=1-k_{\mathcal{M}}$, the nontangential limits of $\kappa$ do not exist on $E$, so Arveson's assertion (A1) fails.

(B) The nontangential boundary values of $k_{\mathcal{M}}$ exist a.e. on $\mathbf{T}^{+}$.

But then, by (10), they must be equal to zero there. Hence $\left.k_{\mathcal{M}}\right|_{\mathbf{T}}=\chi_{\mathbf{T}^{-}}$, the characteristic function of $\mathbf{T}^{-}$. It follows that

$$
\boldsymbol{\kappa}=1-\frac{1}{2 \pi} \int_{0}^{2 \pi} \chi_{\mathbf{T}^{-}}\left(e^{i t}\right) d t=\frac{1}{2},
$$

i.e. $\kappa$ exists but is not an integer. Thus Arveson's assertion (A2) fails.

Further, even the modified definition (8) of $\boldsymbol{\kappa}^{*}$ suffers from the same problem: namely,

$$
\kappa^{*}=1-\lim _{r \nearrow 1} \int_{\mathbf{T}} k_{\mathcal{M}}(r \zeta) d \sigma(\zeta)
$$

and from the existence of the boundary values, equal to 1 , of $k_{\mathcal{M}}$ on $\mathbf{T}^{-}$and the Bounded Convergence Theorem it follows that

$$
\lim _{r \nearrow 1} \int_{\mathbf{T}_{-}} k_{\mathcal{M}}(r \zeta) d \sigma(\zeta)=\frac{1}{2},
$$


while from (10) we have

$$
\liminf _{r / 1} \int_{\mathbf{T}^{+}} k_{\mathcal{M}}(r \zeta) d \sigma(\zeta)=0 .
$$

Thus $\boldsymbol{\kappa}^{*}$ either does not exist, or exists but then must be equal to $\frac{1}{2}$.

Finally, we remark that if we instead of $\Lambda_{+}$consider the portion $\Lambda_{\alpha}$ of $\Lambda$ lying in the sector

$$
\mathbf{C}_{\alpha}:=\left\{r e^{i \theta} \in \mathbf{C}: r \geq 0,0 \leq \theta \leq \alpha\right\},
$$

then we arrive at a $Z$-invariant subspace $\mathcal{M}$ for which $k_{\mathcal{M}}$ has again the properties as above, but with the half-circles $\mathbf{T}^{+}$and $\mathbf{T}^{-}$replaced by the $\operatorname{arcs} \mathbf{T}_{\alpha}:=\mathbf{T} \cap \mathbf{C}_{\alpha}$ and $\mathbf{T} \backslash \mathbf{T}_{\alpha}$, respectively. Consequently, we obtain an example of a pure, $p$-contractive 1-tuple of rank 1 for which $\boldsymbol{\kappa}$ (and $\boldsymbol{\kappa}^{*}$ ) either do not exist, or exist but then are equal to $\alpha / 2 \pi$, i.e. to any given number between 0 and 1 .

\section{References}

[Ag] J. Agler, Hypercontractions and subnormality, J. Operator Theory 13 (1985), 201-217.

[ARS] A. Aleman, S. Richter and C. Sundberg, The majorization function and the index of invariant subspaces in the Bergman spaces, J. Anal. Math. 86 (2002), 139-182.

[AEM] C. Ambrozie, M. Engliš and V. Müller, Operator tuples and analytic models over general domains in $C^{n}$, J. Oper. Theory 47 (2002), 287-302.

[An] N. Aronszajn, Theory of reproducing kernels, Trans. Amer. Math. Soc. 68 (1950), 337404.

[Ar1] W. Arveson, Subalgebras of $C^{*}$-algebras III: Multivariable operator theory, Acta Math. 181 (1998), 159-228.

[Ar2] W. Arveson, The curvature invariant of a Hilbert module over $\mathbf{C}\left[z_{1}, \ldots, z_{d}\right]$, J. Reine Angew. Math. 522 (2000), 173-236.

[Ar3] W. Arveson, The Dirac operator of a commuting d-tuple, J. Funct. Anal. 189 (2002), $53-79$.

[En] M. Engliš, Some problems in operator theory on bounded symmetric domains, Acta Appl. Math. 81 (2004), 51-71.

[GRS] D. Greene, S. Richter and C. Sundberg, The structure of inner multipliers on spaces with complete Nevanlinna Pick kernels, J. Funct. Anal. 194 (2002), 311-331.

[Le] R. N. Levy, Note on the curvature and index of almost unitary contraction operator, preprint math.FA/0007178 (2000).

[MT] S. McCullough and T. Trent, Invariant subspaces and Nevanlinna-Pick kernels, J. Funct. Anal. 78 (2000), 226-249.

[Pa] S. Parrott, The curvature of a single contraction operator on a Hilbert space, preprint math.OA/0006224 (2000). 